Journal of The Magnetics Society of Japan Vol. 13, Supplement, No. S1 (1989)

(C) 1989 by The Magnetics Society of Japan

\title{
AN ANALYSIS OF MULTILAYERED THIN-FILM MAGNETIC FIELD GRADIOMETERS HAVING A SUPERCONDUCTING SPACER
}

\author{
Ronald S. INDECK ${ }^{\circ}$ \\ Department of Electrical Engineering, Washington University \\ St. Louis, Missouri 63130-4899 USA
}

\begin{abstract}
Using a finite element method we have analyzed a multilayered thin-film recording head structure having a thin-film superconducting spacer. We have used this method to obtain simulated output responses of the head to an ideally recorded medium. The results of the analysis for a specific geometry show that the superconducting spacer improves the efficiency of such heads by about $50 \%$. Also, significant improvements are obtained by not completely filling the gap region and by making the superconducting film shorter than the head elements. Gap erosion was found to degrade the performance of these devices severely.
\end{abstract}

\section{INTRODUCTION}

Until recently, the simulation of recording heads necessitated the building of large scale models, making very tedious and simplified analytical analyses, or conducting very laborious numerical simulations. With the advent of faster mini- and micro- computers and the creation of finite element method programs whose pre- and post-processors employ graphical interfaces, much more sophisticated problems can be addressed more easily[1]. These encompass the inclusion of non-linear materials into the simulation or simulating the read process by "sliding" a numerical head model past simulated magnetically recorded bits. We have applied one such program, the Maxwell software from Ansoft Corporation[2] to the design of a superconducting gradiometer.

Recently, gradiometers have been designed and fabricated, in part, to address the growing need for high spatial resolution during read in a magnetic recording system. For example, in a flexible magnetic recording system having an excellent head-medium contact and employing a medium with a large (perpendicular) anisotropy, magnetically recorded densities in excess of $500 \mathrm{kfrpi}$ have been demonstrated[3].

'Supported in part by NSF Grant No. ECS8957148 (Presidential Young Investigator Award)

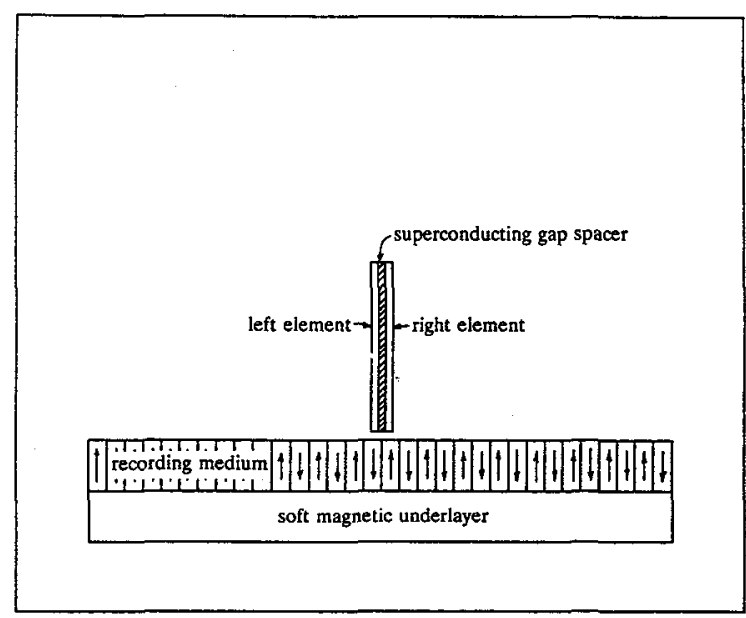

Figure 1. Problem Schematic (not to scale)

Even under near ideal conditions, reading of these ultra-high linear bit densities remains a challenge. The fringing magnetic fields from ultra-high density recordings do not emanate far from the medium making reading difficult. The signal intensities obtained are small due to the relatively small flux available to the read transducer. Moreover, the flux is varying very rapidly in space (corresponding to the extremely high density of magnetization reversals). 
Conventional transducers use relatively large flux concentrating elements (pole pieces) which couple the flux to a pickup coil. Because these elements are large compared to the rapidly varying spatial flux pattern, the efficiency of the head during the read process is small. The efficiency is reduced in part due to the leaking of the flux across the gap region which reduces the amount of flux available at the coil for reading. Several head designs which fill the gap region with conductors and more recently superconducting-films[e.g. 4] have been developed. These heads utilize an effective negative susceptibility in the gap which helps to contain the flux in the pole pieces and increase the coupling (to a coil, magnetoresistive, or Hall device) thereby increasing the head's efficiency. Unfortunately, these designs still use large magnetic elements which will tend to decrease the head's spatial resolution.

In response to this problem, many different multilayered thin-film transducers have been designed which employ thin semiconducting or magnetic films[e.g. 5, 6, 7] whose responses are electrically summed in order to produce a magnetic field gradiometer. These devices may offer greater spatial resolution than the conventional heads but because of their finite throat height, these devices also suffer from imperfect flux linkage of the fringing fields above the medium's transitions. For this reason, we have analyzed a gradiometer structure with a superconducting gap spacer (the schematic is shown in Figure 1).

\section{THE NUMERICAL MODEL}

The model that we use to investigate the performance of the superconducting gradiometers employs a linear finite element method. We have chosen the following problem to analyze:

\section{The Head}

The head consists of two thin-film, highly permeable, isotropic and linear elements. The permeability is chosen to be 500 . The height of the elements is 3 microns. The thickness of the elements is $250 \AA$. The separation (gap) between the pole piece elements is $1000 \AA$ and the head to medium separation is $250 \AA$. The trackwidth is assumed to be infinite. The superconducting thin-film is modeled by a linear permeability of 0.001 .

\section{The Medium}

The recording medium is modeled by a simple permanent magnet material with a permeability of unity; unit permeability was chosen because the medium's magnetization is assumed to be very hard and not perturbed by being in close proximity to the read head. For both the longitudinal and perpendicular recording cases, the magnetization is a square-wave recorded, all 1's pattern. The thickness of the recording layer is $3000 \AA$. For the perpendicular magnetization case, a $3000 \AA$ isotropic soft magnetic underlayer having a permeability of 500 is included.

\section{The Parameters}

The throat height, thickness, and gap erosion parameters of the thin superconducting layer were varied to establish their influence on the head's read performance. In addition, the head was moved over the simulated medium to obtain a calculated output response or "read pulse".

\section{RESULTS AND DISCUSSION}

\section{The Program}

To establish our confidence in the use of the numerical process, we tested the output of the program against several easily analyzed cases. Because the program involves some preprocessing, that is, the making and refining of the mesh, we first investigated the mesh making procedure. Our first task was to determine the discretization errors or artifacts encountered with the mesh making. An infinitesimally small mesh would reduce the discretization effects but this procedure would be prohibitively expensive. For all of our analyses, we chose $1.5 \%$ deviations in output to be tolerable (although most cases exhibited deviations of tenths of a percent or less). We meshed a simple problem several different ways and determined an acceptable method such that minor differences would not 
create noticeable (within our criterion) effects. We found this to be very easy to achieve. With just a few hundred triangles, doubling or tripling the number did not change the outcome. Although, in general, the computation took longer to converge if there were more triangles, in some instances the addition of more triangles seemed to allow for a faster convergence (to the same result).

Since we simulated only a small portion of an actual recording track, we needed to determine how many bits were necessary to approximate an infinite tracklength. Using the three micron high head and 0.5 micron bits, addition of bits after 20 was unnecessary; no change in output was seen as more bits were added, and placing the simulated head in corresponding regions of any of the central bits resulted in similar outputs. For assurance, we used 32 bits in our simulation.

\section{Output}

To determine the output from the heads as described, we integrated the resulting field up the elements and assumed that the elements were linear (non-multidomain) entities. This analysis may be reasonable for a magnetoresistive element linearized for a range of small sensing fields, or certainly for a Hall element (in which case we assumed a unit permeability). There was no simulated sensing or biasing current in the elements; the elements were assumed to be intrinsically linear.

Figure 2 shows the resulting output of the head reading a series of all 1's perpendicular transitions. The graph shows the output of the individual elements and the resulting summed output of the transducers in the gradiometer mode. The output is normalized to the output for two elements without a superconducting gap. It is important to note that the outputs of the two elements are asymmetrical about the centers of the bits. This is due to the fact that the transducers interact; the right transducer has an asymmetric magnetic environment due to the left element and vice versa.

The overall result for the superconducting spacer gives an output about $50 \%$ larger than that without a spacer. This effect is precisely what was anticipated due to the flux expulsion (diamagnetic behavior) of the superconductor. The diamagnetic layer keeps the flux in the pole pieces farther back from the recording surface and up the elements.

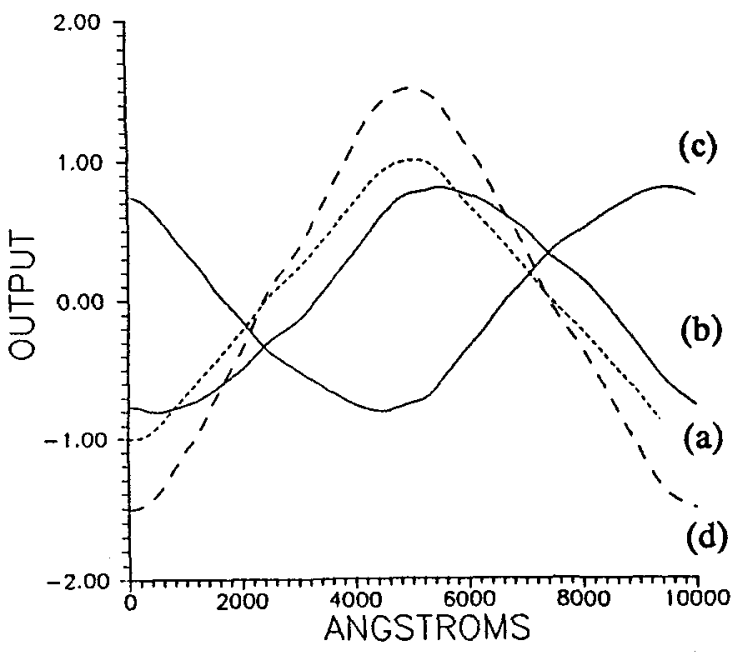

Figure 2. Output vs position for $5000 \AA$ bits:

(a) head with no superconducting spacer,

(b) right element, (c) left element,

(d) head with full superconducting spacer

Next we investigated the effect of filling the gap region only partially with the superconducting material. The results are shown in Figure 3. As the thickness of the superconductor decreases, the relative efficiency of the structure is increased. Symmetrically filling only one quarter of the gap region gives an output $8 \%$ larger than a fully filled gap. An interpretation of this result is suggested by the flux plots of Figures $4 \mathrm{a}$ and $4 \mathrm{~b}$. When the gap is not filled, some of the flux can enter the elements through the inner surface, effectively increasing its permeance. This suggests that when actually fabricating such devices, a nonferromagnetic spacing between the superconducting films and the ferromagnetic members is desirable.

Subsequently, we examined the effect of the height of the diamagnetic spacer; this was done from both ends and can be thought of as 


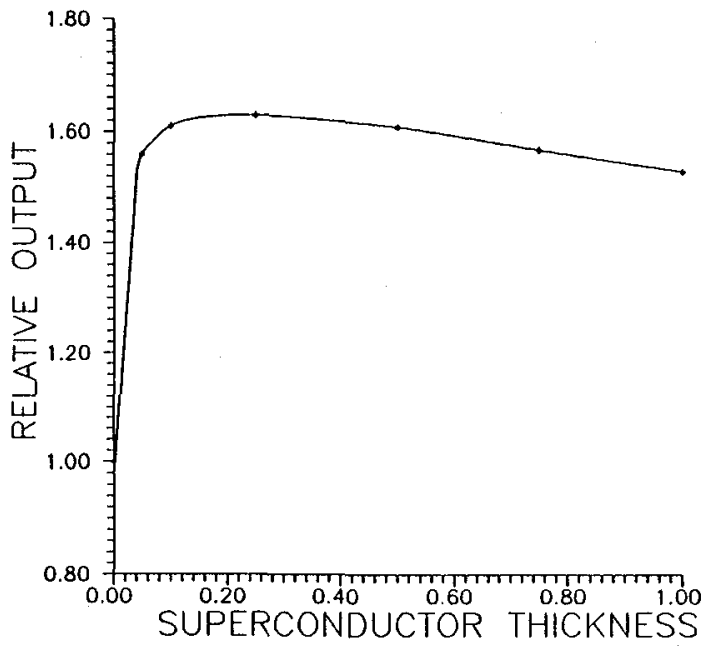

Figure 3. Output vs superconducting spacer thickness for a gap length of $1000 \AA$ (normalized to $1000 \AA$ gap)

creating a back gap and simulating gap erosion respectively. The results can be seen in Figures 5 and 6. The gap erosion in all cases was detrimental to the operation of the device. For deep gap erosion, or for fabrication errors such as a mismatch of the photolithographic masks, the effect is so dramatic that the performance can actually be poorer than that of a head without any superconducting filler. This is believed to be due to the expulsion of the flux from the element -- deep gap erosion creates a preferred path for the flux at the front of the head (see Figure 7).

The inclusion of a back gap (a superconducting filler shorter than the elements) improves confinement of the flux within the element. Some back gap is desirable to allow for a more efficient flux path; if the full gap height has been filled with superconducting film then the flux must travel outside the element ends to close instead of having a lower reluctance path across the gap. A further improvement of almost $50 \%$ again can be achieved with the shortened gap spacer (this gives overall performance improvement of almost $125 \%$ ).
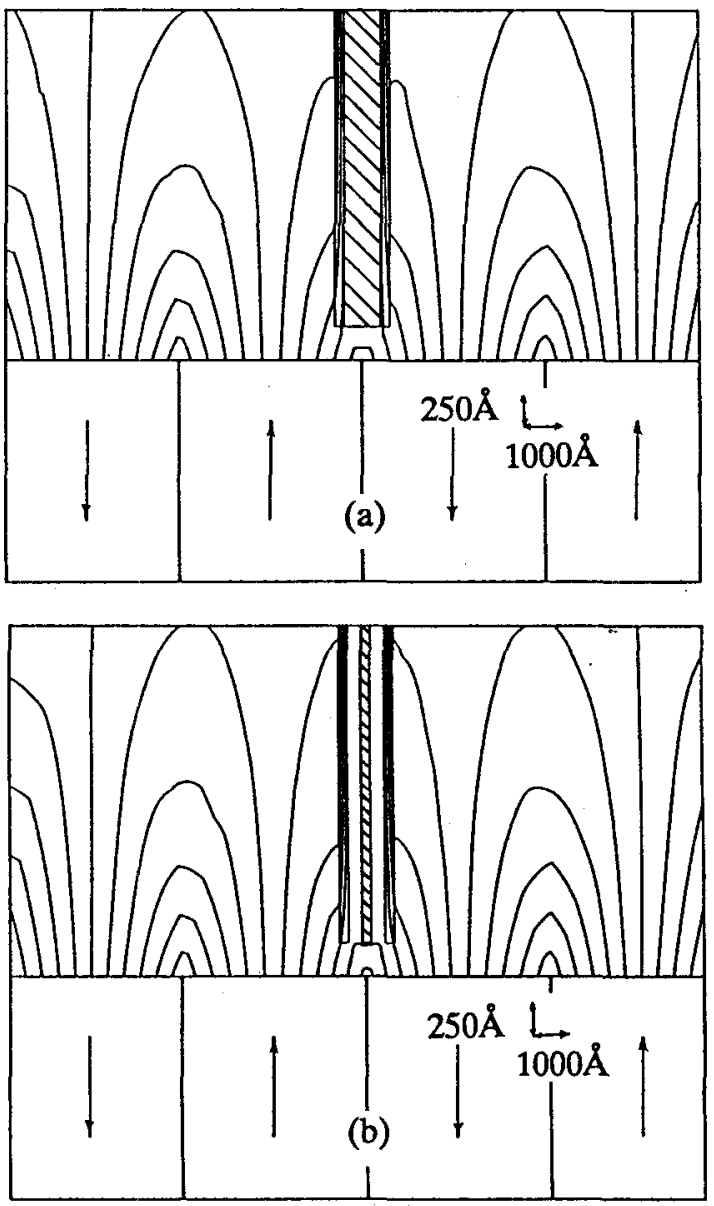

Figure 4. Flux plot for:

a. 100\%-superconductor filled gap

b. 25\%-superconductor filled gap

(inconsistent vertical and horizontal scales)

For the longitudinal recording simulation, similar improvements were found when the highly permeable elements were included; output increases of about $50 \%$ were seen with the superconducting spacer. When the highly permeable elements were replaced with simulated Hall elements (unit permeability), outputs dropped by almost two orders of magnitude. Gains from the superconducting film were much less dramatic, being only a few percent assuming that the field components of 


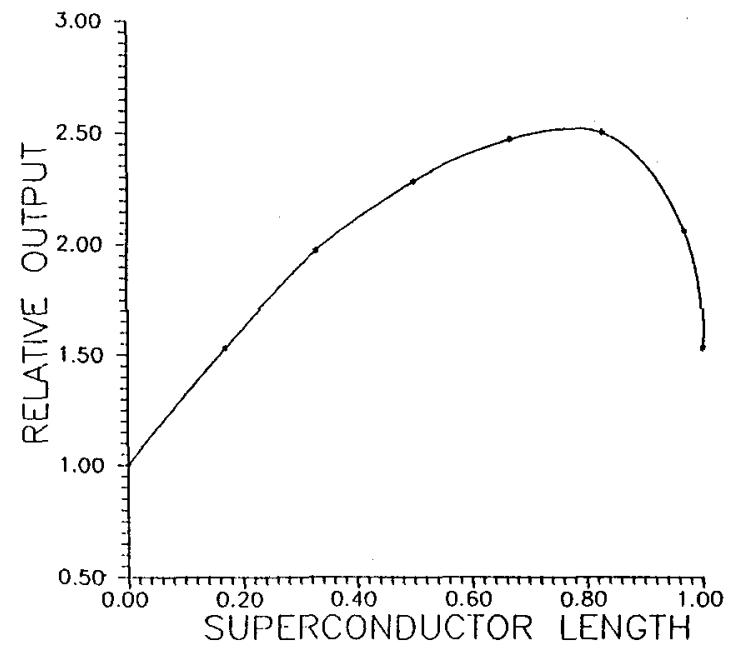

Figure 5. Output vs superconductor length (normalized to 3 micron element length)

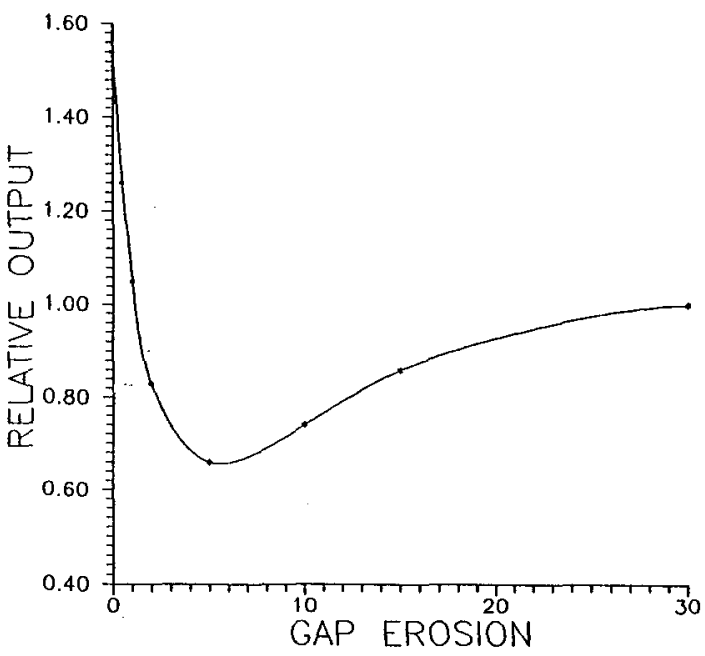

Figure 6. Output vs gap erosion depth (normalized to $1000 \AA$ gap)

interest were normal to the semiconducting films. Without high permeable flux channeling members, inferior results from this head structure were obtained by the simulation.

Some general comment about the computation and the parameters chosen follows.
The permeability of the elements, back layer and the superconductor did not appear to be critical. Changes in permeability from 200 to 2000 or from 0.01 to 0.00001 produced changes in output of less than ten percent. The choice of a unit permeability for the permanent magnet media appears sound, since changes in the medium permeability up to 15 did not significantly affect the outcome.

Changes in element thickness were not seen to be critical -- changing from $250 \AA$ to 500 $\AA$ showed little effect on the read performance at these simulated densities. In addition, gap thicknesses between $1000 \AA$ and $2000 \AA$ were not found to be a critical parameter at $50 \mathrm{kfrpi}$.

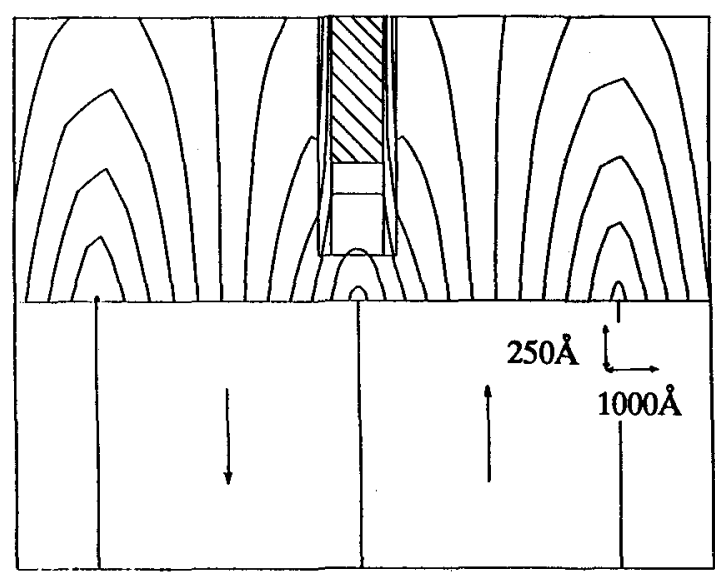

Figure 7. Flux plot for a gap erosion of $0.5 \mathrm{~g}$ (inconsistent vertical and horizontal scales)

\section{Future Considerations}

This simple but helpful analysis employed only linear materials; in particular this meant that there could be no medium remagnetization. Future investigations could include nonlinear materials and medium parameters could be varied more exactly. Although this analysis concentrated on a gradiometer application, other multi-layered thin film structures could be investigated. One obvious candidate would be the conventional thin film head. Additionally, this method could be considered for investigating other recording parameters such as spatial 
sensitivity, perhaps by producing rolloff curves, or by performing peak shift analyses.

\section{CONCLUSIONS}

Based upon a linear finite element analysis of a multi-layered gradiometer head structure with a thin-film superconducting spacer, we find that the diamagnetic properties of the superconducting gap spacer yields an increase in performance of $50 \%$. The performance can be further improved by not completely filling the gap with the diamagnet and significant improvements can be achieved through the shortening of the spacer (creation of a back gap region). Gap erosion severely degrades the performance of a head and can actually be poorer than that of a head without any superconducting spacer for modest erosion (around twice the patterned gap). This simple head geometry works well for longitudinal and perpendicular recording but replacing the highly permeable elements with unit permeability Hall elements was found to give outputs nearly two orders of magnitude smaller.

\section{ACKNOWLEDGEMENTS}

The author is indebted to the members of the Magnetics Research Laboratory at Washington University for their assistance and many fruitful discussions, and to Ansoft Corporation for their support of the Maxwell program.

\section{REFERENCES}

[1] Z. Cendes, IEEE Spectrum, 26, 4, 29-33 (1989).

[2] Ansoft Corporation, Pittsburgh, PA 15213.

[3] S. Yamamoto, et. al., IEEE Trans. Magn., MAG-23, 5, 2070-2072 (1987).

[4] M. Dugas, W. Eidelloth, F. Barnes, IEEE Trans. Magn., MAG-24, 6, 2395-2397 (1988).

[5] G. V. Kelley, et. al., IEEE Trans. Magn., MAG-17, 6, 1890-2 (1981).

[6] R. S. Indeck, J. H. Judy and S. Iwasaki, IEEE Trans. Magn., MAG-24, 6, 2617-2619 (1988).

[7] H. S. Gill, et. al., JAP, 65 (1), $402-404$ (1989). 